\title{
Formation of Internal Modified Line with High Aspect Ratio in Sapphire by Sub-nanosecond Pulsed Fiber Laser
}

\author{
Yasuhiro Okamoto ${ }^{* 1}$, Tomohiro Takekuni ${ }^{* 1}$ and Akira Okada ${ }^{* 1}$ \\ ${ }^{*}$ Graduate School of Natural Science and Technology, Okayama University, \\ 3-1-1 Thsushimanaka, Kita-ku, Okayama 700-8530, Japan \\ E-mail: okamoto@mech.okayama-u.ac.jp
}

\begin{abstract}
The internal modification technique for a sapphire substrate using a sub-nanosecond pulsed fiber laser of 180 ps was experimentally investigated with a normal achromatic focusing lens of $20 \mathrm{~mm}$ focal length, and the possibility of a singulation method with this internal modification technique was discussed. The laser beam of $1060 \mathrm{~nm}$ was focused in sapphire substrate of $0.4 \mathrm{~mm}$ thickness by passing through GaN epitaxial layer grown on the top surface of the sapphire substrate, and the internal modification zone was generated from the stain-finished surface as the bottom surface of sapphire substrate. The laser beam was absorbed from the stain-finished bottom surface, and the absorption point moved in the axis of laser irradiation without moving the focusing optics. The narrow internal modified line less than $5 \mu \mathrm{m}$ in width with a high aspect ratio of more than 30 was successfully obtained only by one laser scanning irradiation from the epitaxial layer side. A sapphire wafer could be broken from the internal modified line with less damage of the epitaxial layer by a stress sufficiently smaller than the tensile strength of sapphire. The breaking strength and its dispersion became smaller with increasing number of laser scans.
\end{abstract}

DOI: $10.2961 /$ jlmn.2014.01.0011

Keywords: sapphire, internal modification, high aspect ratio, ultrashort pulsed laser, subnanosecond pulsed laser, breaking strength

\section{Introduction}

Sapphire has been widely used as a substrate of high brightness LEDs (Light Emitting Diode) because of its excellent physical and electrical properties [1, 2]. The sapphire substrate must be singulated in the manufacturing process of LED devices. Sapphire is hard and brittle, but laser beam processing could be performed without mechanical contact regardless of material hardness. Thus, laser beam processing has been expected as a highthroughput singulation method because of its excellent performance. Various laser beam processing technologies, such as ablation dicing and water-jet dicing, have drawn attention as singulation method, because the laser processing can be carried out regardless of material hardness. Some singulation methods using the laser beam have been developed and widely used in industrial fields $[3,4]$. One of the attractive laser dicing technologies uses an internal modified layer, which is formed by tightly focusing a narrow-emission-bandwidth laser beam inside materials [5-7]. In addition, it was reported that an ultrashort pulsed laser could enhance the extraction efficiency of LEDs, and the short pulsed laser is useful for high quality processing [8]. This laser dicing method normally requires a high-NA (Numerical Aperture) objective lens to achieve the space-selective absorption of laser beam inside a material, and the standoff distance between the processing head and the target material would be narrow. Since it is required to control the distance between the focal point and the target material plane precisely in this method, an alternative technique with highly flexible of focusing control in the process would be useful in industrial applications. Therefore, a highly flexible process for creating a modified layer inside a sapphire substrate was experimentally investigated by using a sub-nanosecond pulsed fiber laser with a wide emission bandwidth and a normal achromatic lens, which can keep a sufficiently wide standoff distance. Moreover, a breaking test was carried out, and its characteristics were also discussed in order to apply this internal modification method to a singulation technique for a sapphire substrate.

\section{Experimental Procedure}

\subsection{Setup of Laser Irradiation Experiment}

Figure 1 shows the schematic diagram of the experimental setup for laser irradiation experiment. A subnanosecond pulsed fiber laser was used as a laser source [9]. MOPA (Master Oscillator Power Amplifier) system of this laser oscillator can control the pulse duration and pulse repetition rate individually by amplifying the seed light of high speed modulated diode laser. The main specifications

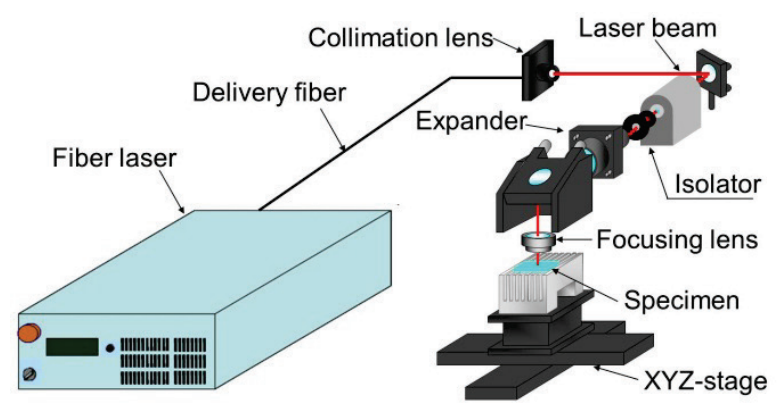

Fig. 1 Schematic illustration of laser irradiation experiment. 
of the laser oscillator are shown in Table 1. The center emission wavelength of the laser beam was $1060 \mathrm{~nm}$, and the pulse duration was set to $t_{p}=180 \mathrm{ps}$ in this study. The laser beam of $1.6 \mathrm{~mm}$ in diameter was expanded fivefold, and then the expanded laser beam of $8 \mathrm{~mm}$ diameter was focused by a normal achromatic lens of $20 \mathrm{~mm}$ focal length. The laser irradiation experiment was carried out in air.

Table 1 Specifications of MOPA pulsed fiber laser.

\begin{tabular}{lc}
\hline Center wavelength $\lambda$ & $1060 \mathrm{~nm}$ \\
Maximum pulse energy $Q$ & $220 \mu \mathrm{J}$ \\
Maximum average power $P_{a}$ & $11 \mathrm{~W}$ \\
Pulse repetition rate $R_{p}$ & $50 \mathrm{kHz}-1 \mathrm{MHz}$ \\
Pulse duration $t_{p}$ & $100 \mathrm{ps}-300 \mathrm{ps}$ \\
\hline
\end{tabular}

Sapphire of $0.4 \mathrm{~mm}$ total thickness was used as a specimen, and a $\mathrm{GaN}$ epitaxial layer of $3 \mu \mathrm{m}$ thickness was grown on the surface, as shown in Fig. 2. The surface of the GaN side was mirror-finished, while its back was stainfinished with $\mathrm{Ra}=0.51 \mu \mathrm{m}$. The main specifications of the sapphire substrate are shown in Table 2. The crystal orientation of wafer was C (0001).

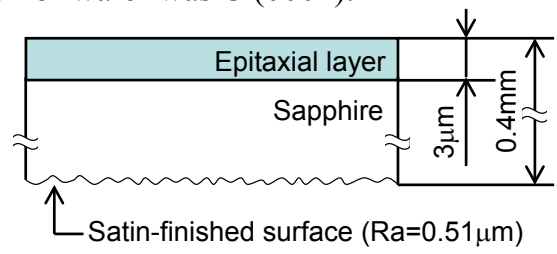

Fig. 2 Schematic illustration of sapphire specimen.

Table 2 Specifications of sapphire substrate.

\begin{tabular}{lc}
\hline Density & $3970 \mathrm{~kg} / \mathrm{m}^{3}$ \\
Specific heat & $0.78 \mathrm{~kJ} /(\mathrm{kg} \cdot \mathrm{K})$ \\
Refractive index & 1.77 \\
Melting temperature & $2050 \mathrm{~K}$ \\
\hline
\end{tabular}

Figure 3 (a) schematically shows the setup of the specimen fixed on the jig with a groove. The bottom side of the specimen was exposed to atmospheric air at the laser scanning line. The GaN epitaxial layer was located on top side of the specimen, and the bottom side of the specimen was stain-finished. Laser irradiation was carried out from the epitaxial layer side of the sapphire substrate. The focal point of the laser beam was adjusted by a fine screw micrometer. Laser scanning was performed either perpendicularly or parallel to the orientation flat line of the sapphire wafer, as shown in Fig. 3 (b).
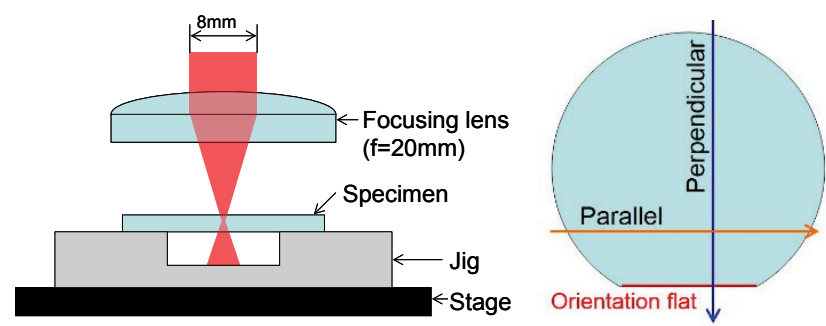

(a) Setup of specimen on jig

(b) Scanning direction

Fig. 3 Setup of specimen and scanning direction

\subsection{Setup of Breaking Test}

In order to measure the breaking strength to separate the sapphire substrate after the laser irradiation, a breaking test was carried out using a tensile testing machine. Each specimen was cut into $5 \mathrm{~mm} \times 10 \mathrm{~mm}$, and a modified layer was formed at the center of the cut specimen. Since it was difficult to clamp a small specimen directly, the specimen was fixed on a glass plate of $1.1 \mathrm{~mm}$ thickness with an epoxy resin adhesive, as shown in Fig. 4 (a). The fixed specimen was used for the evaluation of the breaking strength.

A tensile test was carried out for these specimens by using a clamping jig with a linear guide in order to avoid specimen twisting during the pulling process, as shown in Fig. 4 (b). The pulling direction was perpendicular to the laser scanning line, and the test speed was $0.5 \mathrm{~mm} / \mathrm{min}$. The friction force of the linear guide was subtracted from the maximum load of breakage, and the breaking strength was calculated.

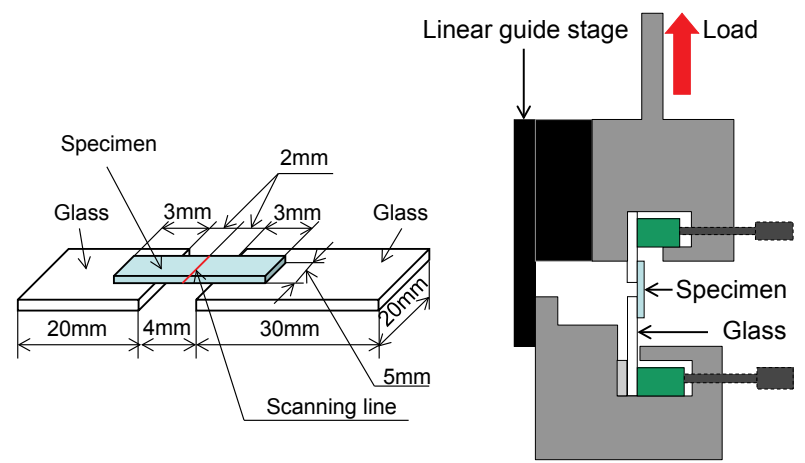

(a) Specimen pasted on glass

(b) Clamping jig

Fig. 4 Setup of breaking test.

\section{Formation Phenomena of Modified Line}

\subsection{Influence of Focal Point on Formation of Modified Line}

The laser scanning experiment was carried for various focal points above and below the surface of the specimen, and the cross sections at the laser scanning line are shown in Fig. 5. These optical micrographs were obtained with an illumination light from the back side of the specimen against an observation camera. The scanning direction was perpendicular to the orientation flat line of the sapphire wafer. The laser irradiation was carried out from the top side of the specimen in the figure, and the focal point was adjusted by a $50 \mu \mathrm{m}$ space. Here, the focal point $z=0$ indicates that the focal point is located on the surface plane of the specimen, and the irradiation direction of the laser beam was considered positive relative to the focal point $z$. The negative focal point $z$ indicates that the focal point is located inside the specimen. The laser beam was mainly absorbed at the top surface of specimen with a focal point of more than $z=-0.15 \mathrm{~mm}$. The material removal was observed at the top surface of specimen around the just focal point, and then the groove depth decreased with the increase in focal point. A distinct modified line with high aspect ratio was formed at the focal points $z=0.20 \mathrm{~mm}$ and $0.25 \mathrm{~mm}$. The height of the modified line was $180 \mu \mathrm{m}$ at $z=-0.20 \mathrm{~mm}$. The internal modified line was grown in the 
coaxial direction of the laser beam only from the bottom side of the specimen at some focal points. However, the Rayleigh length of this optical setup was approximately 20 $\mu \mathrm{m}$ at $1060 \mathrm{~nm}$, even if the refractive index of sapphire was considered. The length of this modified line was $180 \mu \mathrm{m}$, which is much larger than Rayleigh length. The bottom side of the specimen was the stain-finished, and the bottom surface roughness was larger than the glossy top surface roughness. When the laser irradiation direction was set from the bottom side of the specimen in Fig. 3(a), the laser beam was absorbed only at the stain-finished bottom side of the specimen regardless of the focal point. Thus, the laser beam scattered at the roughened surface of bottom side, and the absorption rate of laser energy increased. Therefore, it is considered that the formation of the modified line could be achieved only from the bottom side of the specimen.

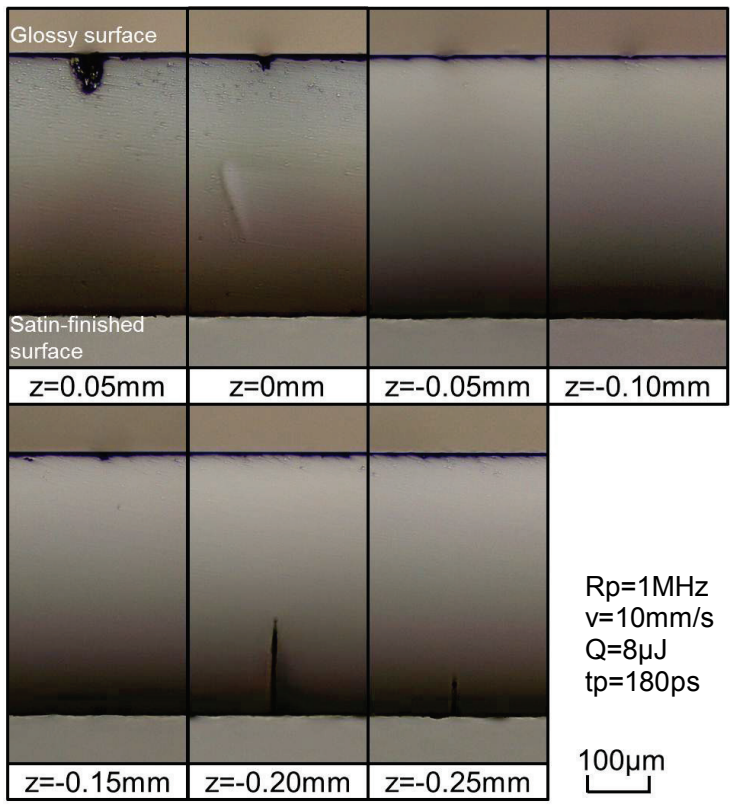

Fig. 5 Cross sections of specimen at laser scanning line for various focal points.

\subsection{Generation Behavior of Internal Modified Line}

In order to discuss the formation process of this internal modified line with high aspect ratio, the observation of the laser irradiation area during the scanning experiment was carried out using a high-speed video camera (Shimadzu, Hyper Vision HPV-2A) with a zoom lens. Figure 6 shows the captured images observed perpendicularly to the laser irradiation direction. The laser irradiation was carried out from the top side and moved from the right to left in these images. The specimen was cut to $1.0 \mathrm{~mm}$ in width, and both surfaces of the observation plane were polished. A halogen lamp illumination was set behind the specimen, and observation was carried out from the opposite side of illumination. The time " 0 " indicates the start of observation. Since the frame rate of the video camera was synchronized with the pulse repetition rate of $R_{p}=125 \mathrm{kHz}$, every laser pulse could be captured in each video frame. The luminous area in the image is the absorption point of the laser pulse, and the black line is the internal modified line. After the luminous point reached the top region of the

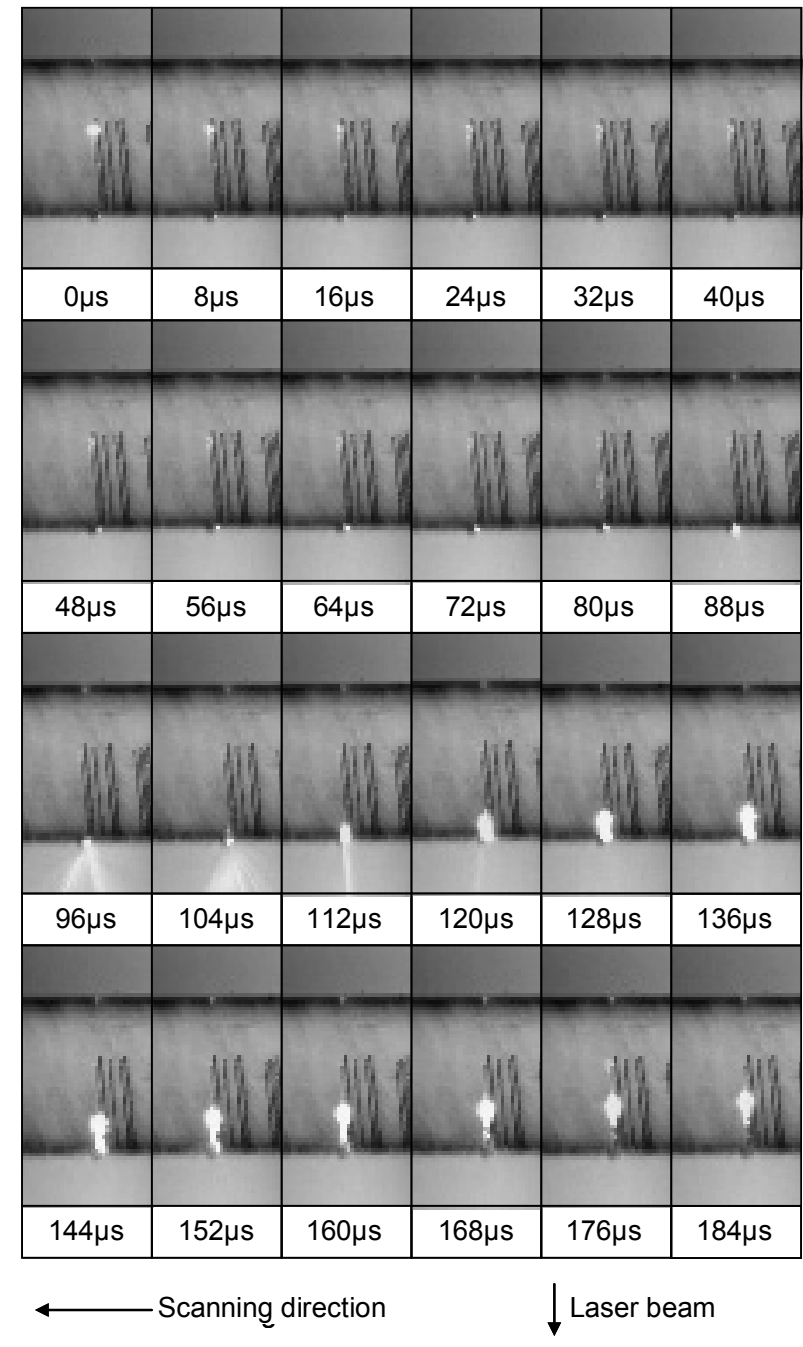

$\mathrm{Rp}=1 \mathrm{MHz}, \mathrm{v}=10 \mathrm{~mm} / \mathrm{s}, \mathrm{Q}=8 \mu \mathrm{J}, \mathrm{tp}=180 \mathrm{ps}, \mathrm{z}=-0.20 \mathrm{~mm} \quad 100 \mu \mathrm{m}$

Fig.6 Movement of absorption point without movement of focal position in laser irradiation direction.

internal modified line, this absorption point suddenly moved down to the bottom side of the specimen, and climbed up and slightly moved toward the scanning direction without moving the focusing optics in the irradiation axis of the laser beam. The absorption point followed the scanning and irradiation axes of the laser beam, and the movement of the absorption point was repeated.

On the other hand, the emission bandwidth of this fiber laser is more than $50 \mathrm{~nm}$, as shown in Fig. 7. In general, a narrow emission bandwidth and a high-NA objective lens are effective for inducing the nonlinear absorption of laser energy inside a transparent material. However, Rayleigh length inevitably decreased with a use of a high-NA lens. Tight focusing could enhance the nonlinear absorption, and the laser energy could be absorbed by nonlinear interactions such as multi-photon absorption [10]. In contrast, a relatively small-NA and a wide emission bandwidth were used in this experiment, and the nonlinear absorption was unstable when the focal point was located in the middle of the specimen. The pulse duration was $t_{p}=180 \mathrm{ps}$, and the peak power was relatively high. 


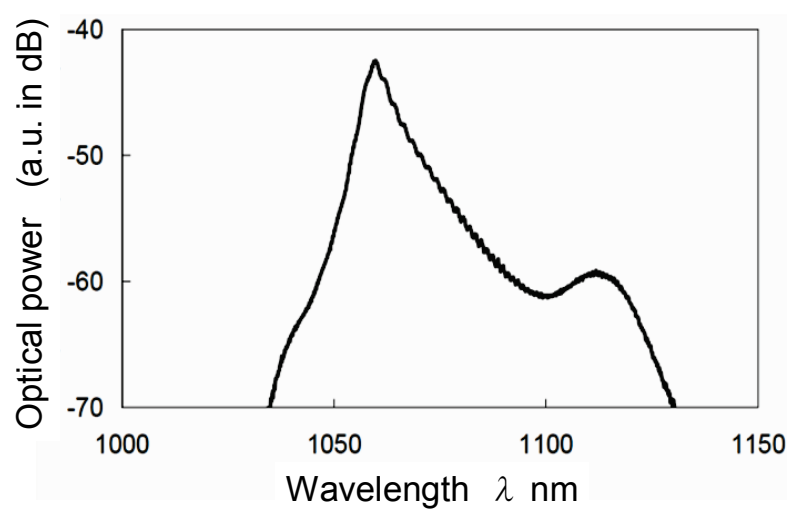

Fig. 7 Emission spectrum of sub-nanosecond pulsed fiber laser.

The transmission ratio of the laser beam of the specimen with the stain-finished surface was $13.5 \%$ lower than that of the specimen with both sides polished one, when the measurement of transmitted power was carried out by using this laser source. In addition, it was reported that a high pulse repetition rate increases the laser absorption energy, and it was suggested that seed electrons for avalanche ionization are provided mainly by thermally excited free electrons at locations apart from the focus at high pulse repetition rates [11]. Although this pulse energy was insufficient for inducing nonlinear absorption at the focal point, the laser beam was absorbed at the roughened interface between the sapphire wafer and the air. After the absorption of laser energy at the bottom side of the specimen, the absorption point of the laser moved in the reverse direction of the laser irradiation along the axis of the laser beam owing to the slightly tight focusing of the laser beam and the large Rayleigh length with the wide emission bandwidth of this fiber laser. It is considered that the stain-finished surface could increase and stabilize the absorption of laser energy at the bottom side of the specimen. Since the absorption of laser energy was induced when the laser intensity was higher than the threshold, the absorption area was limited in the center region of the laser beam. In addition, the large Rayleigh length led to the formation of a wide absorption region in the irradiation direction. Therefore, these phenomena resulted in the stable formation of a narrow modified line with high aspect ratio. It is considered that the combination of a wide emission spectrum with high pulse repetition rate and a slightly roughed surface could lead to the unique formation of this modified line.

\subsection{Appearance of Modified Line}

In order to analyze the modified line, the laser modified specimen was polished perpendicularly to the scanning line by an argon ion beam method, and the modified line was observed by SEM (Scanning Electron Microscope). Figure 8 (a) shows the whole view, and the oblique lines in the figure are marks left by the argon ion beam process. Areas A and B in Fig. 8 (a) are magnified in Figs. 8 (b) and (c), respectively. As shown in Fig. 8 (b) of magnified view at area $\mathrm{A}$, the width of the modified line is $5 \mu \mathrm{m}$, and the aspect ratio of this modified line is 36 . The absorption of the laser beam could not be confirmed at the top surface and the interface between the epitaxial layer and the sapphire substrate. Obvious damage could not be observed

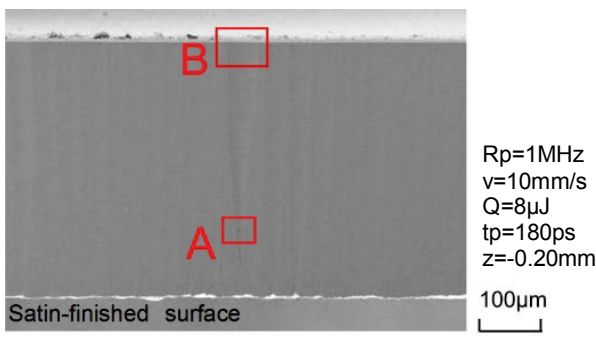

(a) Whole view

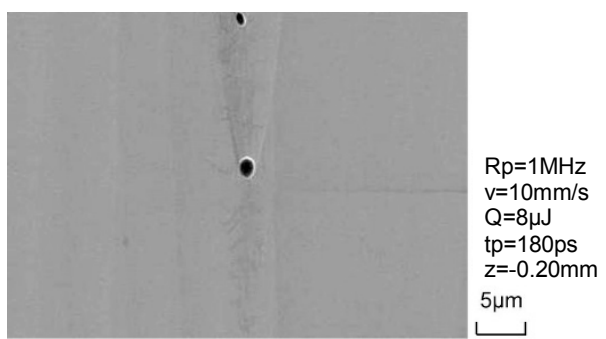

(b) Magnified view of internal modified area A

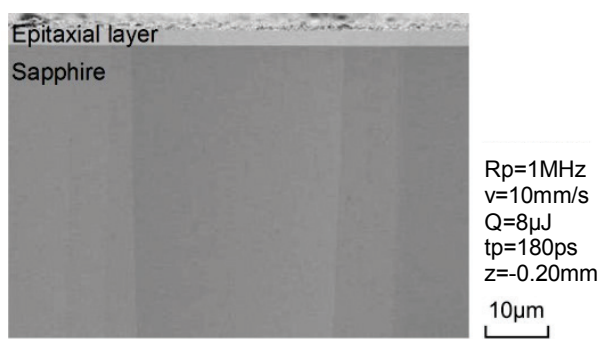

(c) Magnified view of interface between epitaxial layer and sapphire substrate at area B

Fig. 8 Cross sections of internal modified line.

in the epitaxial layer of GaN, as shown in Fig. 8 (c). Cracks and voids remained inside the modified line, and it is considered that the temperature increased more than the evaporation point at the modified line. This indicates that the energy of the laser beam was absorbed in the modified line.

Raman analysis was carried out to discuss the crystal state of the internal modified area. In order to increase the area of the internal modified line, the specimen was tilted and polished. Then, the modified areas, which were encircled by red lines, were observed as discontinuous lines in the microphotograph in Fig. 9 (a). When the Raman shift of sapphire was measured by using an argon laser of 514.5 $\mathrm{nm}$ wavelength, the Raman shift spectrum of sapphire showed the strongest peak at $418 \mathrm{~cm}^{-1}$.

The full width half maximumu FWHM of the peak at $418 \mathrm{~cm}^{-1}$ was measured at the area of $20 \mu \mathrm{m}$ by $50 \mu \mathrm{m}$ in Fig. 9 (a). Figure 9 (b) shows the distribution of the FWHM of the Raman peak at $418 \mathrm{~cm}^{-1}$ measured by a spot at $0.7 \mu \mathrm{m}$ diameter. The Raman FWHM at $418 \mathrm{~cm}^{-1}$ in the area of the modified line became larger than that in the area of the non-modified area. In general, increasing the FWHM of a Raman peak indicates the deterioration of the crystalline state [12]. In addition, the peak shift of the Raman spectrum was also detected in this modified area by laser irradiation. The change in crystal structure led to the variation in stress filed. Therefore, it is considered that this modified line has the possibility as starting point of cleavage for singulation process. 


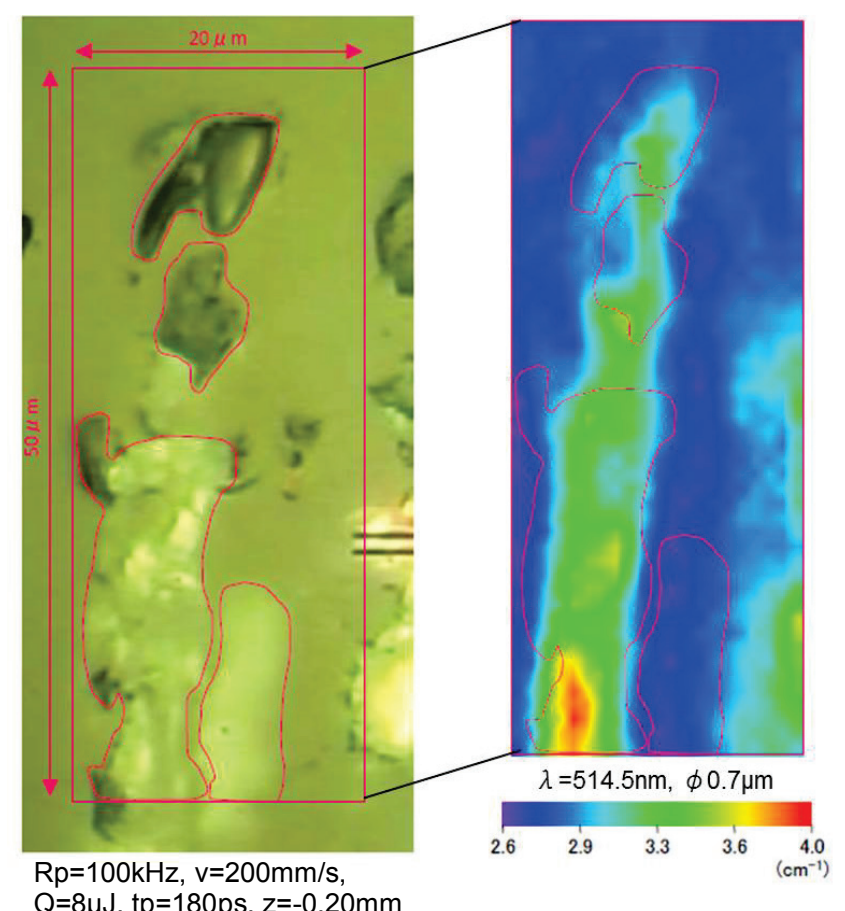
$\mathrm{Q}=8 \mu \mathrm{J}, \mathrm{tp}=180 \mathrm{ps}, \mathrm{z}=-0.20 \mathrm{~mm}$

(a) Microphotograph

(b) Distribution of Raman FWHM at $418 \mathrm{~cm}^{-1}$

Fig.9 Raman analysis of internal modified line.

\section{Influence of Laser Irradiation Conditions on Formation of Modified Line}

Figure 10 shows cross sections of the modified line for various scanning velocities at pulse repetition rates of $R_{p}=100 \mathrm{kHz}$ and $1 \mathrm{MHz}$. Laser irradiation was carried out from the epitaxial layer side, and the scanning direction was perpendicular to the orientation flat line of the sapphire wafer. The focal point was adjusted to $z=-0.20 \mathrm{~mm}$ when the highest modified line was obtained, as shown in Fig. 5. As shown in Fig. 10, the modified line could be successfully formed inside the sapphire substrate from the bottom side of the specimen even at a higher scanning speed of $v=200 \mathrm{~mm} / \mathrm{s}$.

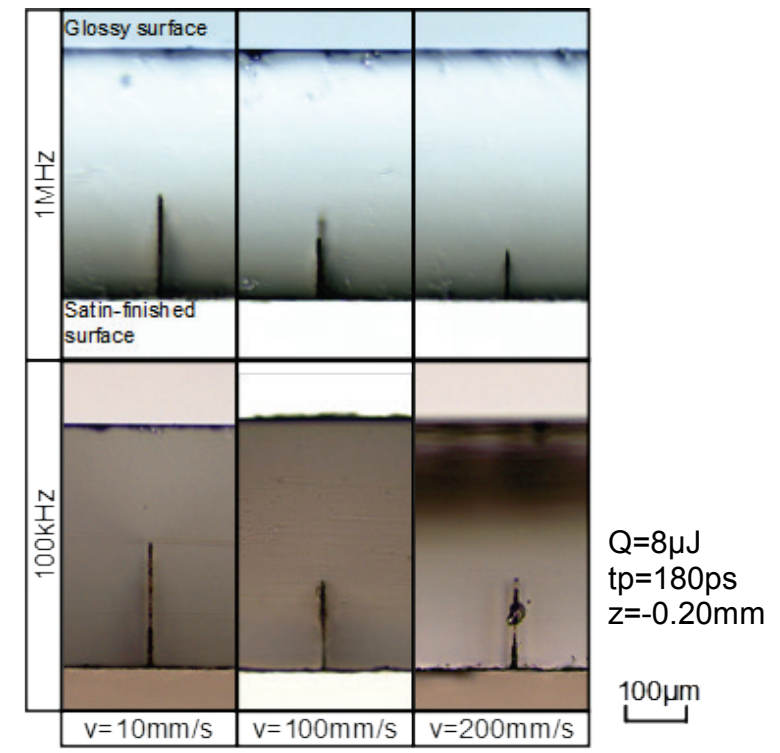

Fig. 10 Cross sections of modified line for various scanning conditions at pulse repetition rate $100 \mathrm{kHz}$ and $1 \mathrm{MHz}$.
Figure 11 shows the relationships between height of the modified line and the scanning velocity at pulse repetition rates of $R_{p}=100 \mathrm{kHz}$ and $1 \mathrm{MHz}$. The height of the modified line became larger at the low pulse repetition rate of $R_{p}=100 \mathrm{kHz}$, and the higher modified line could be obtained at a higher pulse energy. On the other hand, the height of the modified line increased with decreasing scanning velocity, and a little difference in height of the modified line was observed between scanning speeds of $v=$ $100 \mathrm{~mm} / \mathrm{s}$ and $200 \mathrm{~mm} / \mathrm{s}$. It is suggested that the formation of this modified line is related to the pulse energy. The high aspect ratio of the modified line was more than 20 even at a high scanning velocity of $v=200 \mathrm{~mm} / \mathrm{s}$, and highthroughput processing could be expected.

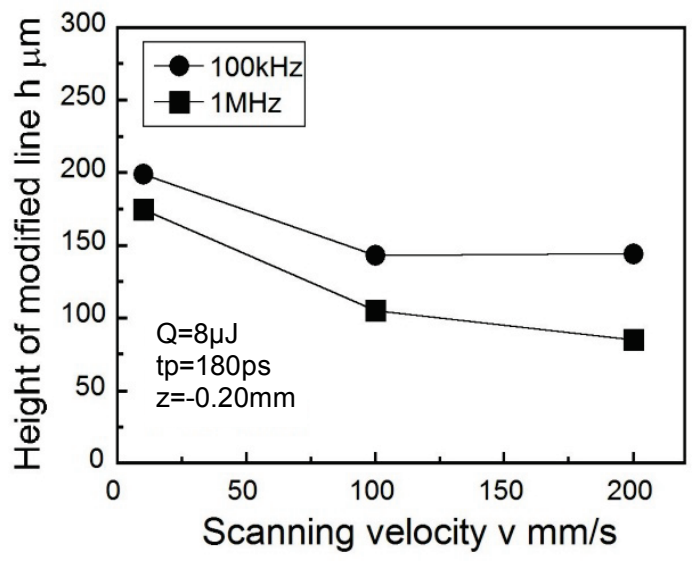

Fig. 11 Height of modified line for various scanning velocities

\section{Breaking Test}

\subsection{Influence of Crystal Orientation on Breaking Strength}

The breaking strength for cleaving the sapphire substrate that propagated from the internal modified layer was investigated by setting the laser scanning line to be either perpendicular or parallel to the orientation flat line of the sapphire wafer, in which the crystallographic plane was (1120). Evaluation results of breaking strength, which was determined by averaging five measurements, are shown in Fig. 12. All specimens were scanned under the same laser irradiation conditions: scanning velocity $v=10 \mathrm{~mm} / \mathrm{s}$, pulse repetition rate $R_{p}=1 \mathrm{MHz}$, pulse energy $Q=8 \mu \mathrm{J}$, pulse duration $t_{p}=180 \mathrm{ps}$ and focal point $z=-0.20 \mathrm{~mm}$. The breaking strength with scanning perpendicular to orientation flat line (crystallographic plane (1010)) became lower than that with scanning parallel to orientation flat line (crystallographic plane $(11 \overline{2} 0)$ ). The coefficients of thermal expansion were different in the directions of parallel and perpendicular to the $\mathrm{C}$ axis, but they were the same in both scanning directions. However, a little difference in breaking strength was observed in both scanning directions. Sapphire is a monocrystalline material, and the hexagonal structure of crystal might affect the difference of breaking strength. There was a little difference in breaking strength due to the crystal orientation. The breaking strengths in both scanning directions could be significantly lower than the tensile strength of sapphire 2,250 $\mathrm{MPa}$. The singulation of the 
sapphire substrate could be performed by a sufficiently smaller stress with a little difference in breaking strength by crystal orientation.

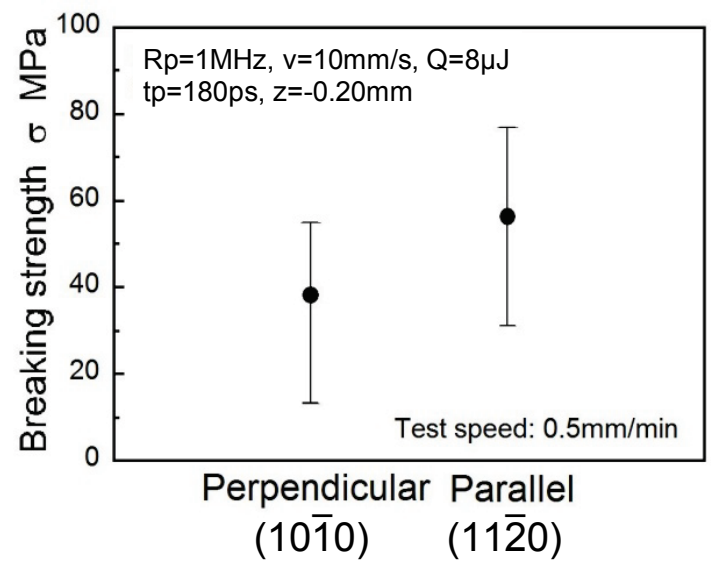

Fig. 12 Breaking strength of specimens scribed parallel and perpendicular to orientation flat.

\subsection{Influence of Number of Laser Scans on Breaking Strength}

In order to investigate the influence of number of laser scans, the laser irradiation experiments were carried out with one and two laser scans under the same laser irradiation conditions. Measurement results of breaking strength with one and two laser scans are shown in Fig. 13. The scanning direction was perpendicular to the orientation flat line of the sapphire wafer, and the focal point was the same for one and two laser scans. The breaking strength measured with two laser scans was lower than that measured with one laser scan, and its dispersion became smaller with increasing number of laser scans. A reliable singulation process could be expected by increasing number of laser scans.

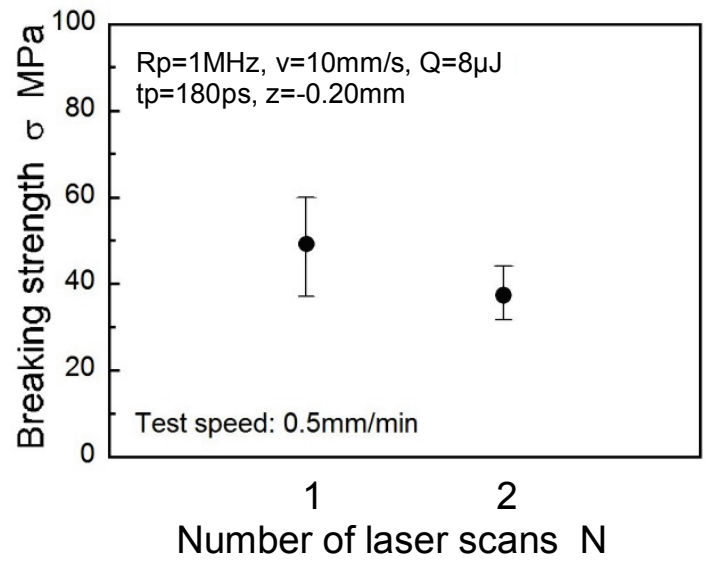

Fig.13 Breaking strength of sapphire with one scan and two laser scans.

\section{Appearance of Cleavage Surface}

Figure 14 shows cleavage surfaces with one and two scans after the breaking test. As shown in the figure, the modified line was not formed continuously but discontinuously at a certain distance. A columnar modified layer could be observed from the bottom side of satin- finished surface, and the height of the modified line was one-third of the specimen thickness under this laser irradiation condition. In the case of one laser scan, the long curved cleavage lines, which would be due to crack propagation, were generated at the cleavage plane from the tip of the modified line. On the other hand, the cleavage lines due to crack propagation were short and straight in the case of two laser scans. The cleavage plane obtained with two laser scans was superior to that with one laser scan, since the columnar modified line was formed more densely by increasing the number of laser scans.

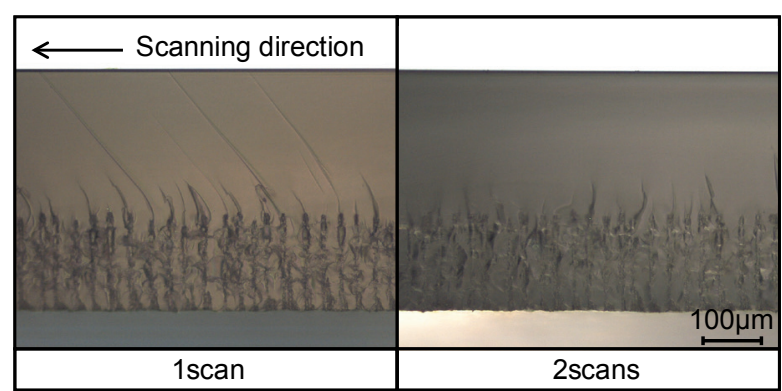

$\mathrm{Rp}=1 \mathrm{MHz}, \mathrm{v}=10 \mathrm{~mm} / \mathrm{s}, \mathrm{Q}=8 \mu \mathrm{J}, \mathrm{tp}=180 \mathrm{ps}, \mathrm{z}=-0.20 \mathrm{~mm}$

Fig.14 Influence of pulse repetition rate on shear stress.

Figure 15 shows the SEM photograph of cleavage surface at the bottom edge of the sapphire substrate. The columnar modified lines from the bottom side were grown by moving the scanning point. The higher modified line was formed by the strong absorption of laser energy, which led to the generation of voids, as shown in Fig. 6. After the formation of the highest modified line, the height of the modified line dropped markedly, and this growth and drop were repeated periodically. The modified line was grown up from the bottom side again after the formation of the highest one. Cleavage propagated from this modified line, and the sapphire substrate could be successfully separated.

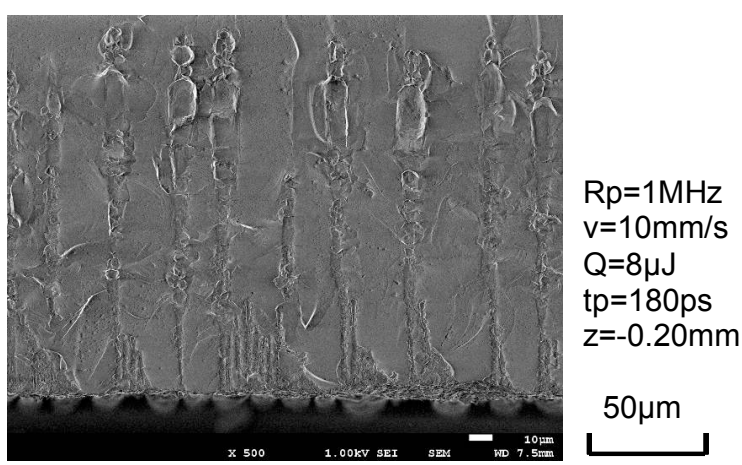

Fig.15 Cleavage surface at the bottom edge of sapphire substrate.

Figure 16 shows the SEM photograph of the cleavage plane at the interface between the epitaxial layer and the sapphire substrate. The cleavage plane of the sapphire substrate was like a mirror surface, and the epitaxial layer could be cleaved with the sapphire substrate. Fatal damage could not be observed at the epitaxial layer, and the sapphire substrate could be divided from the modified line without fatal damage to the epitaxial layer.

The sapphire substrate with a GaN epitaxial layer of 3 $\mu \mathrm{m}$ thickness could be divided from the internal modified 
line by a sufficiently small tensile stress without fatal damage. This internal modification technique using a subnanosecond pulsed fiber laser with a wide emission spectrum and a normal focusing lens could be highly expected as the singulation method for a sapphire substrate with high throughput.

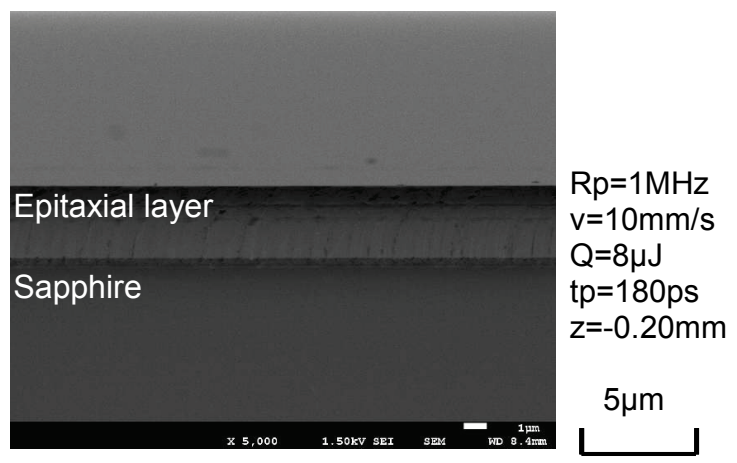

Fig.16 Cleavage plane at interface between epitaxial layer and sapphire substrate.

\section{Conclusions}

An internal modification technique for a sapphire using a sub-nanosecond pulsed fiber laser was experimentally investigated with a normal achromatic focusing lens. The main conclusions obtained in this study are as follows:

(1) An internal modification with high aspect ratio could be formed from a stain-finished surface as the bottom side of the specimen by laser irradiation from the top side of a $\mathrm{GaN}$ epitaxial layer using a normal achromatic focusing lens and a sub-nanosecond pulsed fiber laser.

(2) The laser beam was absorbed from the stain-finished surface as the bottom side of the specimen, and the absorption point moved in the axis of laser irradiation without moving the focusing optics.

(3) A narrow internal modified line, less than $5 \mu \mathrm{m}$ in width with a high aspect ratio of more than 30 could be obtained only by one laser scan.

(4) The sapphire substrate could be divided with a sufficiently small stress by the modified line at the bottom side, and the epitaxial layer grown on the top side of the sapphire substrate was cleaved without fatal damage.

(5) The breaking strength and its dispersion could be reduced by increasing the number of laser scans.

(6) The length of cleavage line due to crack propagation was short, and the internal modification technique has a possibility of singulation method for sapphire wafers.

\section{Acknowledgments}

The authors would like to thank Sumitomo Electric Industries, Ltd. for their contribution of laser source. The authors would also like to express sincere gratitude to $\mathrm{Mr}$. Tabuchi, Technical Assistant of Department of Mechanical Engineering, Okayama University for his useful assistance in the preparation of experiment.

\section{References}

[1] S. W. Chung, Y. S. Zhao, C. H. Lin and H. P. Lee: The Investigation of InGaN MQW Electroabsorption Modulator using the LED/Modulator/Detector Monolithically Integrated Structure, Proceedings of Materials Research Society Symposium, Vol.639, (2001) G13.3.1.

[2] I. Ferguson, M. Schurman and I. Eliashevich: Recent Developments in the Growth of GaN-Based Compound Semiconductors, Journal of the Korean Physical Society, Vol.39, (2001) S433.

[3] D. Karnakis, E. K. Illy, M. H. Knowles, E. Gu, M. D. Dawson: High-throughput Scribing for the Manufacture of LED Components, Proceedings of SPIE, Vol.5366, (2004) 207.

[4] T. Nilsson, F. Wagner and B. Richerzhagen: Scribing of GaN Wafer for White LED by Water-jet-guided Laser, Proceedings of SPIE, Vol.5366, (2004) 200.

[5] M. Kumagai, N. Uchiyama, Ohmura E., Sugiura R., K. Atsumi and K. Fukumitsu: Advanced Dicing Technology for Semiconductor Wafer-Stealth Dicing, IEEE Transactions on Semiconductor Manufacturing, Vol.20, No.3, (2007) 259.

[6] E. Ohmura, F. Fukuyo, F. Fukumitsu and H. Morita: Internal Modified-layer Formation Mechanism Into Silicon With Nanosecond Laser, Journal Achievement in Material and Manufacturing Engineering, Vol.17, (2006) 381.

[7] M. Nagasawa: Laser Dicing Technology of Disco, Proceedings of 73rd Laser Material Processing Conference, (2010) 173. (in Japanese)

[8] J. H. Lee, N. S. Kim, S. S. Hong and J. H. Lee: Enhanced Extraction Efficiency of InGaN-Based Light-Emitting Diodes Using 100-kHz FemtosecondLaser-Scribing Technology, IEEE Electron Device Letters, Vol.31, No.3, (2010) 213.

[9] M. Kakui, S. Tamaoki, Y. Kaneuchi, H. Kohda, Y. Tanaka, Y. Okamoto, Y. Uno and B. Baird: Pulsed Fiber Laser "SumiLas" for Micro-processing, SEI Technical Review, Vol.72, (2011) 127.

[10] M. D. Perry, B. C. Stuart, P. S. Banks, M. D. Feit, V. Yanovsky and A. M. Rubenchik: Ultrashort-pulse Laser Machining of Dielectric Materials, Journal of Applied Physics, Vol.85, No.9, (1999) 6803.

[11] I. Miyamoto, K. Cvecek and M. Schmidt: Evaluation of Nonlinear Absorptivity and Absorption Region in Fusion Welding of Glass Using Ultrashort Laser Pulses, Physics Procedia, Vol.12, (2011) 381.

[12] M Becker, H. Scheel, S. Christiansen, H. P. Stunk: Grain orientation, texture, and internal stress optically evaluated by micro-Raman spectroscopy, Journal of Applied Physics, Vol.106, (2007) 063531.

(Received: August 30, 2013, Accepted: January 26, 2014) 